\title{
DENDROGEOMORPHOLOGICAL ANALYSIS OF MASS MOVEMENT \\ A TECHNICAL NOTE ON THE RESEARCH METHOD
}

\author{
R.R. Braam, E.E.J. Weiss and P.A. Burrough, Utrecht
}

\section{SUMMARY}

Dating of mass movement events by examining the course of eccentricity of the annual rings of trees, inclined as a result of these movements, can be a problem in the case of multiple inclined trees showing compound trunk curvature and complex reaction wood. These problems, however, can be solved when sampling of ring width cores is based on accurate analysis of the form of the trunk.

\section{INTRODUCTION}

A new, automated method for studying mass movement variability using ring width data gahered from inclined trees, has been presented elsewhere (BRAAM et al. 1987) in this Journal. This paper deals with some technical problems that are involved in dendrogeomorphological analysis of mass movement in the case of multiple inclined trees showing two or more different inclination directions in the trunk. Multiple inclined trees can show very complex growth forms as a result of compound trunk curvature when

ISSN 0341-8162

(C) 1987 by CATENA VERLAG,

D-3302 Cremlingen-Destedt, W. Germany

$0341-8162 / 87 / 5011851 /$ US\$ $2.00+0.25$ the directions of successive tilts were different and the periods between the tilts were long (decades). The ring width pattern of the annual growth rings will, as a result of eccentric growth with largest ring width in the direction of inclination, also be complex. As will be shown, this complexity does not hinder analysis of eccentricity as long as sampling of cores is based on analysis of the form of the trunk.

\section{INCLINED TREES, REACTION WOOD, TRUNK CURVATURE AND ECCENTRICITY}

Inclination of a tree causes growth of reaction wood in the trunk and consequent eccentricity of the annual rings. As a result of the reaction wood growth, the inclined trunk curves gradualy upward so that vertical growth can be maintained. This curvature is caused by the pressure (coniferous trees) or tension (deciduous trees) that develops alongside the trunk by reaction wood growth (WESTING 1965). In case of coniferous trees reaction wood is formed on the lower side of the inclined trunk and is called compression wood, in case of deciduous trees it develops on the upper side of the inclined 


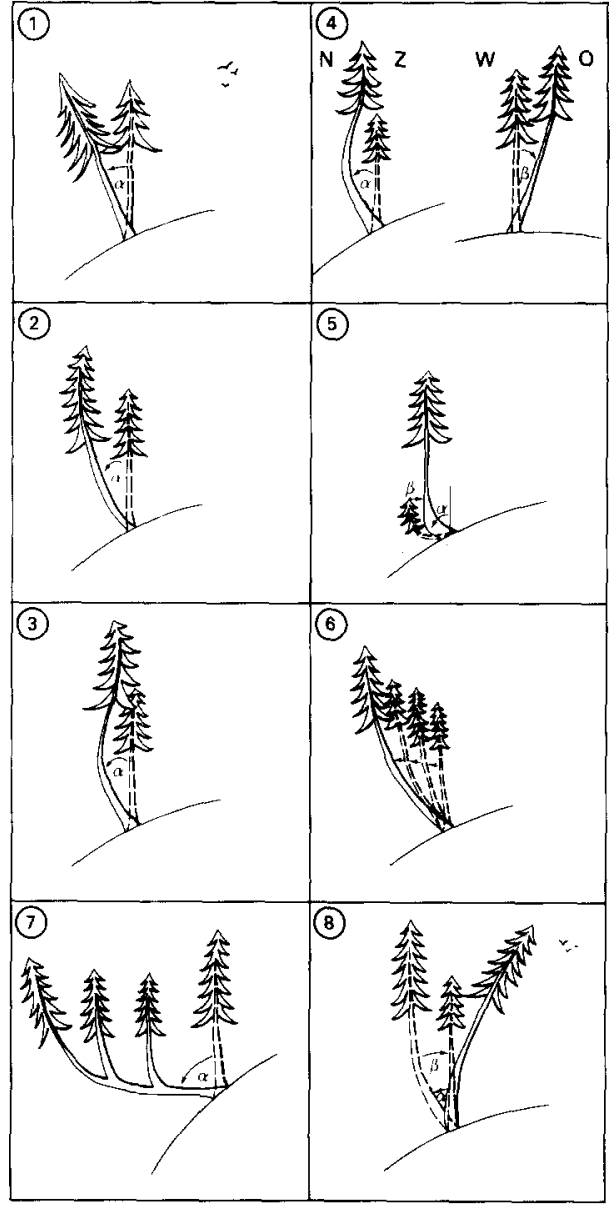

Fig. 1: Schematic trunk forms that can develop in a tree as a result of one or more tilting occurences. Bends are the result of warped growth, caused by reaction wood that develops in non-vertical parts of the trunk..

trunk and is called tension wood. In both cases annual ring growth changes from concentric to eccentric. The reaction wood of conifers is yellowish and reddish brown and consists of short, thick walled cells and annual rings are widest at the lower side of the trunk. Reaction wood of deciduous trees is yellowish or gray and consists of long dense cells and the rings are widest at the upper side of inclined trunks (SHRODER 1980).

In fig. 1 several schematic forms of the trunk are shown that can develop as a result of one or more tilting occurences. Besides curvature of the trunk, caused by reaction wood growth, the form of the trunk changes also as a result of the normal upward growth of the stem tip after tilting. The gradual development of an s-form, which is typical for single inclined trees, is described in detail by ALESTALO (1971). The different stages are shown in fig.1, nos.1 to 3. Multiple inclined trees show one or more of these basic forms when viewed from different positions, as is shown in fig.1, no. 4. The typical "pistol-butted" form results from severe inclination in the youth of the tree (fig.1, no.5). Gradual tilting results in a form equal to that resulting from single tilting (stage 2, fig.1, no. 2), but in this case the stem tip is never vertical. Extreme tilting causes often loss of apical dominance (fig.1, no. 7). Fig.1, no. 8 shows a tree form resulting from successive tilts in opposite direction.

Analysis of the form of the trunk can reveal the (minimum) number of tilting occurences, the directions of tilt and their relative age. The main points of attention are

1. amount of inclination of the trunk as a whole,

2. the number of bends, their direction and relative position in the trunk,

3. the amount of inclination of the upper part of the trunk (stem tip),

4. apical dominance.

It should be noted that two bends in opposite direction are the normal result 

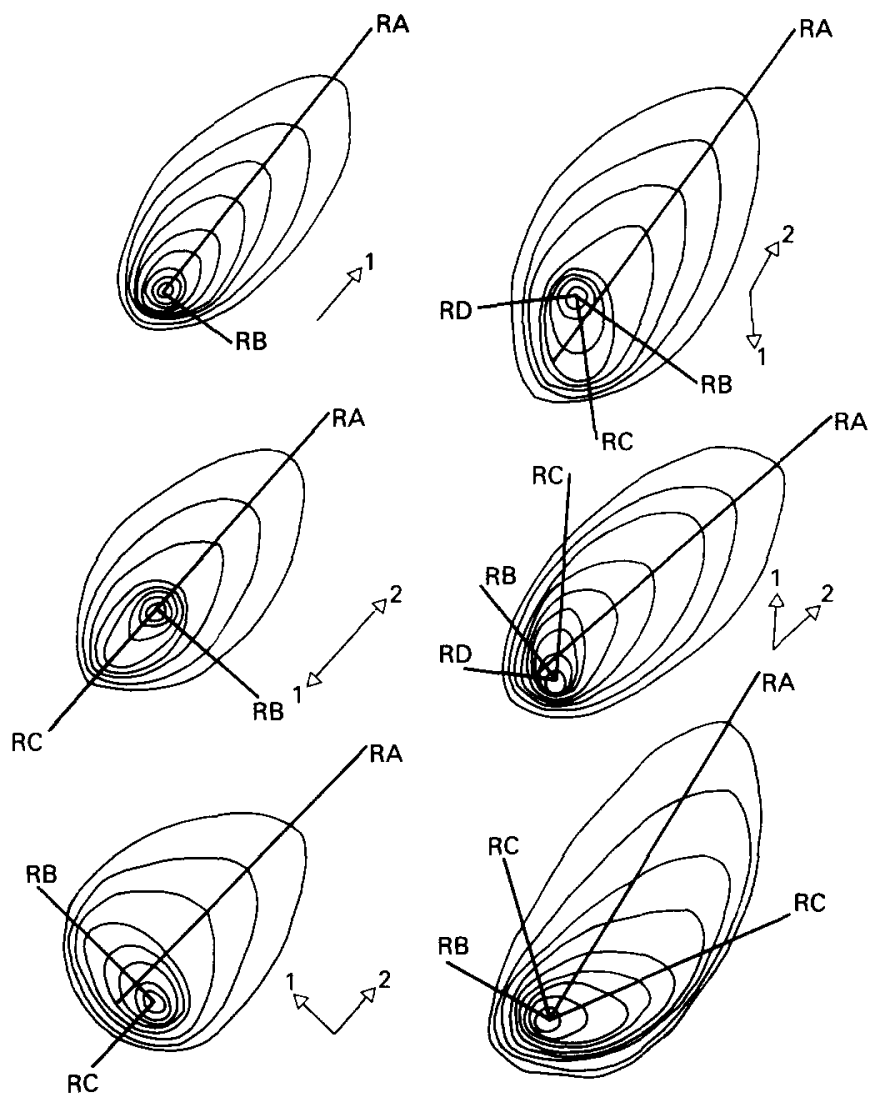

Fig. 2: Cross section of the trunk at the bases of inclined trees. Rings are widest in the direction of inclination. Arrows indicate (successive) tilting directions, straight lines indicate optimal radii for core sampling.

of a single, not recent, tilt (see fig.1, nos. 3 and 4). Warping of the trunk is generally easier in young (thin) trees than in older (thick) trees, and will be restricted to the upper part of the trunks in the older trees. For trees tilted at very young age it will be possible to regain an entirely vertical and straight trunk. In such cases analysis of the form of the trunk reveals nothing about the tilting event. This will also be the case for a tilting event that is succeeded by another tilt within only a few years. Analysis of the form of the trunk reveals thus only a minimum number of tilts.

The precise number and dates of tilts can be revealed by analyzing the course of eccentricity as a measured alongside, at least two, radii at right angles to each other, or from a cross-section of the trunk. When only cores are sampled (this will mostly be the case) accurate dating can be a problem when sampling is not based on analysis of the form of the trunk. When, however, cores are taken for each inclination direction (one 

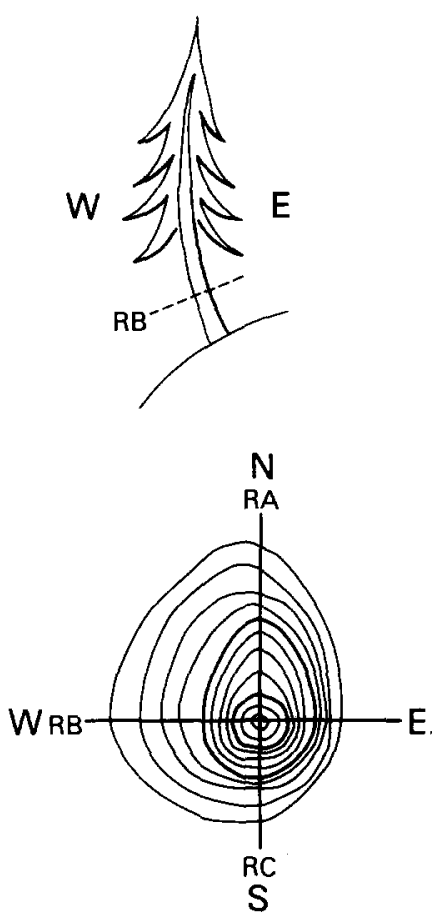

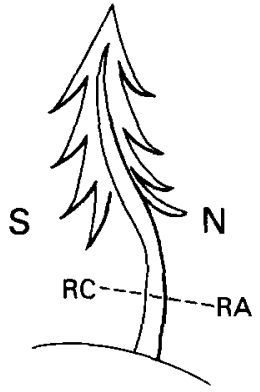

$E_{\text {RA-RB }}=\frac{R A-R B}{R A+R B}$

$E R B-R C=\frac{R B-R C}{R B+R C}$

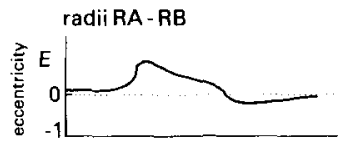

radii $R B-R C$
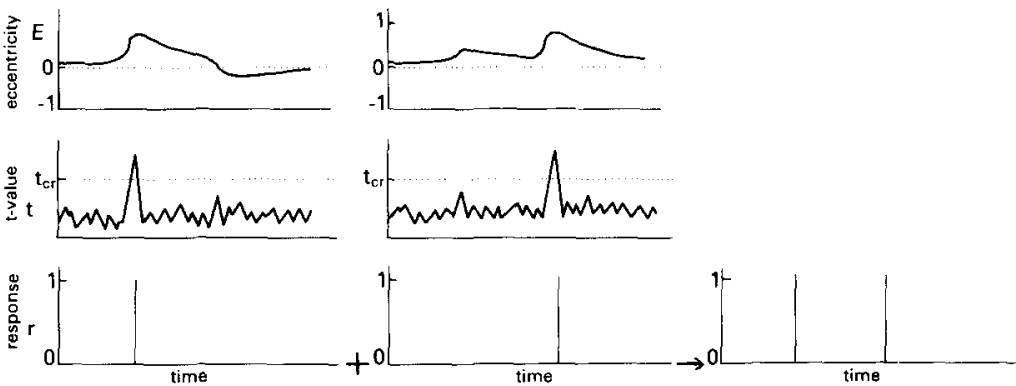

Fig. 3: Sampling and analysis of eccentricity for a doubly inclined tree with different directions of tilting. Eccentricity values for radii pairs $R A-R B$ and $R B-R C$ are shown. Changes of the level of eccentricity are analyzed using a double moving window and a t-test (see BRAAM et al. 1987).

Significant level changes $\left(t>t_{r r}\right)$ date inclination events. The results for the two pairs are summed to establish a record of inclination events for the tree $(R)$. 
in a direction opposite to that of the inclination and the other at squared angles) there is no problem. Eccentricity values should be computed for each pair of cores, and the achieved eccentricity series should be analyzed separately (see for analysis method BRAAM et al. 1987 elsewhere in this Journal). The dating results can simply be summed, although double dates for the same year should be skipped.

Fig.2 schematically shows some ring width patterns that could be the result of one or more tilting events in different directions, and shows sampling directions.

Fig.3 illustrates how sampling could occur to solve the problems involved in dating multiple inclined trees, and the way in which analysis results are combined.

In this case three cores are necessary for adequate analysis of eccentricity. Eccentricity series should be computed and anlyzed for radii RA and RB (revealing the inclination and date of tilting in northern direction) and for radii $\mathrm{RB}$ and $\mathrm{RC}$ (revealing the inclination in western direction). When the different tilting directions are not at squared angles to each other, as in contrast to this example, four cores would have been necessary.

In general, two cores should be taken for each direction of inclination for adequat analysis of eccentricity.

\section{CONCLUSIONS}

The problem of dating accurately the tilting events of multiple inclined trees, showing complex curvature of the trunk, can be solved in a simple way by analyzing the form of the trunk and using two cores (taken at squared angles to each other) for each observed direction of inclination.

\section{REFERENCES}

ALESTALO, J. (1977): Dendrochronological interpretation of geomorphic processes. Soc. Geogr. Tenn. 105-140.

BRAAM, R.R., WEISS, E.E.J. \& BURROUGH, P.A. (1987): Spatial and temporal analysis of mass movements using dendrochronology. CATENA 14, 573-584.

SHRODER, J.F. (1980): Dendrogeomorphological analysis of mass movement on Table Cliffs Plateau Utah. Quaternary Research 9, 168-185.
Address of authors:

R.R. Braam, E.E.J. Weiss \&

P.A. Burrough

Department of Physical Geography, University of Utrecht

Utrecht, The Netherlands 